\title{
Dynamic Behaviors of a Moored Floating Structure with Flexible Skirts
}

\author{
Wen-Kai Weng ${ }^{1}$, Duc-Tru Tran ${ }^{2}$ and Ruey-Syan Shih $^{3}$ \\ 1. Research Center for Ocean Energy and Strategies, National Taiwan Ocean University, Keelung 202, Taiwan, R.O.C. \\ 2. Ph.D. Program in Department of Harbor and River Engineering, National Taiwan Ocean University, Keelung 202, Taiwan, R.O.C. \\ 3. Department of Construction and Spatial Design. Tungnan University, New Taipei City 222, Taiwan, R.O.C
}

Received: November 27, 2015 / Accepted: December 09, 2015 / Published: January 31, 2016.

\begin{abstract}
A numerical model was developed by using the dual boundary element method to investigate the dynamic behavior of a moored floating structure with a pair of vertical and flexible skirts attached at its bottom in the linear wave field. Theoretical conception is based on potential theory with linear external forces. The motions of the structure were assumed to be small and linear. The flexible skirts mounted beneath the structure were assumed uniform flexural rigidity and the thickness of the skirts was negligible. Comparison between the present model and Gesraha's solution was made to verify the results for a moored floating structure with or without rigid skirts. The influence of the skirt rigidity on the moored floating structure, moored lines and waves is investigated in this study. The results show that, the natural frequencies of structure's oscillation, moored force, wave reflection and transmission tend to the region of short-period waves when the flexible rigidity gradually decreases. Positive correlation exists between the aft mooring force and the pitch motion of the floating structure.
\end{abstract}

Key words: Floating structure, dual boundary element method, response amplitude operators, flexible skirts.

\section{Introduction}

In recent decades, floating structures are increasingly used in near shore regions to protect against wave energy or to control shoreline erosion. With the development of various types and the advantage of easy installation, the floating structures offer engineers another suitable choice to design a relatively inexpensive structure for local environments or for special requirements. Though the floating structures have many excellent applications for improvement in the coastal environmental improvement, they are often preferred in relatively low wave energy regions.

Many papers have discussed the character and efficiency of floating structures, not only in various analytic theories but also in the improvement of structure type. Murali, et al. [1] discussed the reflection

Corresponding author: Wen-Kai Weng, professor, research fields: floating body, coastal structure and wave power conversion. and transmission characteristics of cage floating breakwaters through the use of experimental manners. Isaacson, et al. [2] showed the responses of floating breakwater and compared the results with experimental and field data. Sannasiraj, et al. [3, 4] utilized the two-dimensional finite element method to analyze the mooring forces and the responses of a single floating pontoon-type breakwater, and this method has also been adopted to evaluate hydrodynamic coefficients and the responses in sway, and heave of multiple floating structures. Lee and Cho [5] investigated the numerical study of how the incident wave interacted with a moored pontoon-type floating breakwater. Kashiwagi [6] presented research on the wave-exciting forces and wave-induced motion of a floating body in two-layer fluids with finite depth. Huang and Tang [7] studied the wave-body interaction of floating dual pontoon structures in a $2 \mathrm{D}$ fully nonlinear numerical wave tank based on the BIEM (boundary integral 
equation method). Wang and Sun [8] experimentally studied a novel floating breakwater fabricated with large numbers of diamond shaped blocks.

On the other hand, some authors have devoted their research to develop various forms of thin breakwaters. Williams, et al. $[9,10]$ carried out the effects of the various wave and structural parameters on the efficiency of the breakwater as a barrier to wave action. His work has also considered the dynamics of a flexible floating breakwater which extended the entire water depth, and the dynamics of a submerged compliant breakwater consisting of a flexible, beamlike structure anchored to the seabed and kept under tension by a small buoyancy chamber at the tip. The reflection and transmission of incident waves interacting with a long rectangular breakwater with two thin sideboards protruding vertically downward was studied by Gesraha [11]. He also studied the exciting forces, added-mass and damping coefficients, responses of the structure, and the transmission coefficient. Wang and Ren [12] presented the reflection and transmission of small amplitude waves by a flexible, porous and thin beamlike breakwater in the seabed.

In this study, a floating structure with vertical flexible skirts was improved by the character of the floating structure. A numerical model was developed for analyzing the behaviors of a floating structure by using the DBEM (dual boundary element method) and a suitable Green function analyzing the flexible skirts in this paper. The RAOs (response amplitude operators) of the structure, wave reflection and transmission coefficients, and forces on mooring line were calculated and analyzed for different rigidity of skirt with the whole range of a dimensionless parameter $k a$.

\section{Theoretical Development}

\subsection{Problem Definition}

The definition sketch of the analyzed region is shown in Fig. 1. A moored floating structure of a rectangular cross section consists of two flexible skirts and is situated on the sea at a uniform depth $h$.
Cartesian coordinates are employed with the z-axis directed vertically upwards from its origin on the undisturbed free surface. The structure is located symmetrically at $\mathrm{x}=0$, and linked on the sea floor by an idealized mooring system. Motions of the structure are assumed to be small and linear, when the structure is subjected to a train of small amplitude waves of a height of $S_{0}$ and frequency $\sigma$ traveling in the negative $\mathrm{x}$-direction. The usual assumptions of fluid of analytic region are inviscid and incompressible. Under the above assumptions made, motion of fluid will be irrotational and can be described in terms of velocity potential:

$$
\Phi(x, z, t)=\frac{g \varsigma_{0}}{\sigma} \varphi(x, z) e^{i \sigma t}
$$

and the potential of analytic region must satisfy Laplace's equation.

$$
\nabla^{2} \varphi(x, z)=0
$$

The analytic region was divided into three sub-regions further to simplify the problem. Those sub-regions are each termed as Region I $\left(x \geq \ell_{1}\right)$, Region II $\left(-\ell_{2} \leq x \leq \ell_{1}\right)$ and Region III $\left(x \leq-\ell_{2}\right)$. Region II includes the floating structure; the inner fluid motion of this region will contain a scattered and radiated effect. Regions I and III are set at the positions far away from the structure and are assumed to be beyond the disturbance caused by the floating structure's motions; this sets two auxiliary boundaries $x=\ell_{1}$ and $x=-\ell_{2}$. The potential of Regions I and III can be described in the following form, respectively.

$$
\begin{gathered}
\varphi_{1}(x, z)=\left(e^{i k\left(x-\ell_{1}\right)}+K_{r} e^{-i k\left(x-\ell_{1}\right)}\right) \cdot \frac{\cosh k(h+z)}{\cosh k h} \\
\varphi_{3}(x, z)=K_{t} e^{i k\left(x+\ell_{2}\right)} \cdot \frac{\cosh k(h+z)}{\cosh k h}
\end{gathered}
$$

where, $K_{r}$ and $K_{t}$ are the reflected coefficient and transmitted coefficient. $k$ is the incident wave number which is the root of the linear dispersion relationship $\sigma^{2}=g k \tanh k h$ where $g$ is the acceleration of gravity. The potentials of Regions I and III, combined with their normal derivatives at auxiliary boundaries 


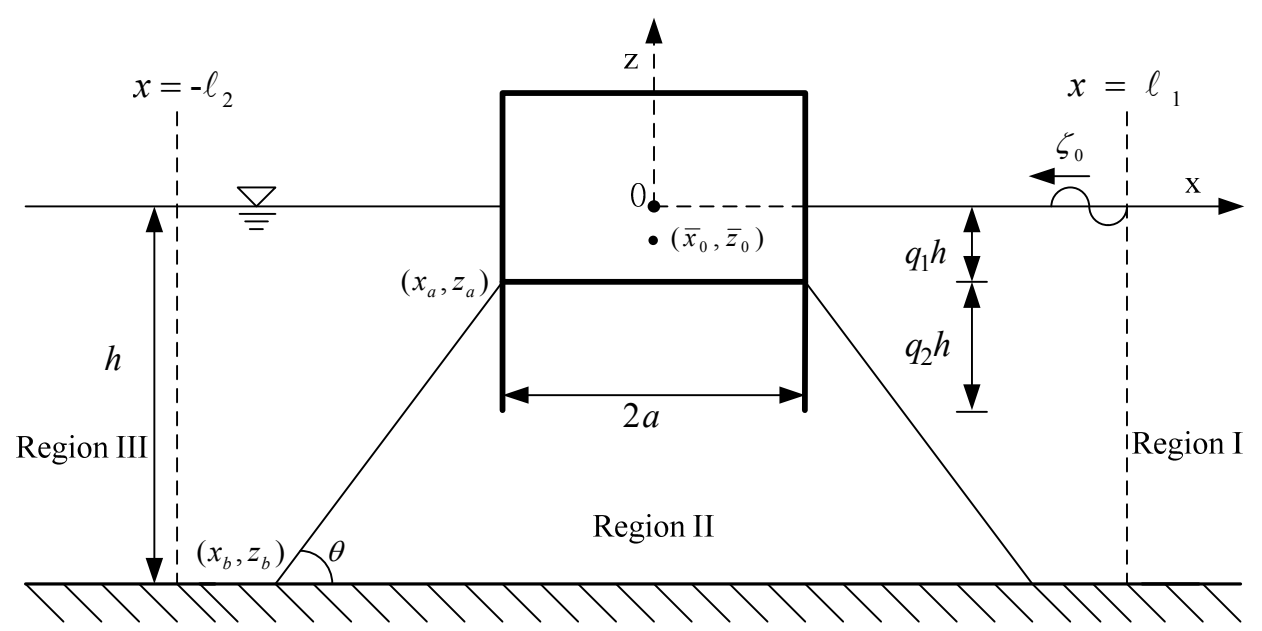

Fig. 1 Definition sketch.

$x=\ell_{1}$ and $x=-\ell_{2}$, are expressed as:

$$
\begin{gathered}
\varphi_{1}\left(\ell_{1}, z\right)=\left(1+K_{r}\right) \frac{\cosh k(h+z)}{\cosh k h} \\
\bar{\varphi}_{1}\left(\ell_{1}, z\right)=i k\left(1-K_{r}\right) \frac{\cosh k(h+z)}{\cosh k h} \\
\varphi_{3}\left(-\ell_{2}, z\right)=K_{t} \frac{\cosh k(h+z)}{\cosh k h} \\
\bar{\varphi}_{3}\left(-\ell_{2}, z\right)=-i k K_{t} \frac{\cosh k(h+z)}{\cosh k h}
\end{gathered}
$$

\subsection{Boundary Condition}

The boundary condition on the auxiliary requirements of continuity of mass and energy flux across the fluid interfaces between each region imply the following matching condition:

$$
\begin{gathered}
\varphi_{1}\left(\ell_{1}, z\right)=\varphi_{2}\left(\ell_{1}, z\right) \\
\bar{\varphi}_{1}\left(\ell_{1}, z\right)=\bar{\varphi}_{2}\left(\ell_{1}, z\right) \\
\varphi_{3}\left(-\ell_{2}, z\right)=\varphi_{2}\left(-\ell_{2}, z\right) \\
\bar{\varphi}_{3}\left(-\ell_{2}, z\right)=\bar{\varphi}_{2}\left(-\ell_{2}, z\right)
\end{gathered}
$$

The boundary condition on the free surface and the impermeable sea floor are subject to the following equations, respectively.

$$
\begin{gathered}
\bar{\varphi}_{2}(x, z)=\sigma^{2} /\left(g \cdot \varphi_{2}(x, z)\right), \quad z=0 \\
\bar{\varphi}_{2}(x, z)=0, \quad z=-h
\end{gathered}
$$

On the floating structure boundary, for the analysis of structure response, it is assumed that, the structure behaves as a two-dimensional rigid structure and the structure will undergo small amplitude surge, heave and pitch motions when it is in response to the incident and diffracted waves. The displacement for those three mode motions may then be expressed as:

$$
\begin{aligned}
& \hat{X}=x_{0}-\bar{x}_{0}=\xi \cdot e^{i \sigma t} \\
& \hat{Z}=z_{0}-\bar{z}_{0}=\eta \cdot e^{i \sigma t} \\
& \delta=\omega \cdot e^{i \sigma t}
\end{aligned}
$$

In which $\left(\bar{x}_{0}, \bar{z}_{0}\right)$ is a coordinate of the center of mass of the structure at rest and $\left(x_{0}, z_{0}\right)$ is its instantaneous position. $\xi, \eta$ and $\omega$ are amplitude of surge, heave and pitch motions, respectively.

On the surface of the floating structure, the displacement for the floating structure may be expressed as:

$$
\begin{gathered}
\bar{X}=\left(x_{0}-\bar{x}_{0}\right)-\delta\left(z-\bar{z}_{0}\right) \\
\bar{Z}=\left(z_{0}-\bar{z}_{0}\right)+\delta\left(x-\bar{x}_{0}\right)
\end{gathered}
$$

The first order kinematic boundary on immersed surface of structure may be written as:

$$
\begin{aligned}
& \frac{\partial \Phi}{\partial n}=\frac{\partial\left(x_{0}-\bar{x}_{0}\right)}{\partial t} \frac{\partial x}{\partial n}+\frac{\partial\left(z_{0}-\bar{z}_{0}\right)}{\partial t} \frac{\partial z}{\partial n} \\
& +\frac{\partial \delta}{\partial t}\left[\frac{\partial x}{\partial n}\left(z-\bar{z}_{0}\right)+\frac{\partial z}{\partial n}\left(x-\bar{x}_{0}\right)\right]
\end{aligned}
$$

The boundary condition on the flexible skirt is slightly different from the floating structure; the velocity of the skirt's motion is added to the dynamic 
boundary condition, and assuming the vertical component of the skirt's velocity is small and ignoring, and the variation of the angular velocity by skirt's motion is small too.

On the flexible skirts, the displacement for the floating structure may be expressed as:

$$
\begin{gathered}
\bar{X}=\left(x_{0}-\bar{x}_{0}\right)-\delta\left(z-\bar{z}_{0}\right)+U(z, t) \\
\bar{Z}=\left(z_{0}-\bar{z}_{0}\right)+\delta\left(x-\bar{x}_{0}\right)
\end{gathered}
$$

Therefore, the first order kinematic boundary on the flexible skirts of structure attaches skirts may be written as:

$$
\begin{aligned}
& \frac{\partial \Phi}{\partial n}=\frac{\partial\left(x_{0}-\bar{x}_{0}\right)}{\partial t} \frac{\partial x}{\partial n}+\frac{\partial\left(z_{0}-\bar{z}_{0}\right)}{\partial t} \frac{\partial z}{\partial n}+\frac{\partial U(z, t)}{\partial t} \frac{\partial x}{\partial n} \\
& +\frac{\partial \delta}{\partial t}\left[\frac{\partial x}{\partial n}\left(z-\bar{z}_{0}\right)+\frac{\partial z}{\partial n}\left(x-\bar{x}_{0}\right)\right]
\end{aligned}
$$

where, $U(z, t)=v(z) e^{i \sigma t}$ is the displacement amplitude of the flexible skirts, $n$ is the boundary normal vector.

\subsection{Equation of Structure's Motions}

The hydrodynamic force on the floating structure acted up by fluid pressure and external forces, the equation can be calculated by integrated the pressure around the wetted floating structure surface as:

$$
\begin{gathered}
m \cdot \frac{\partial^{2} \hat{X}}{\partial t^{2}}=\int_{S_{b}} p \frac{\partial x}{\partial n} \mathrm{~d} s-K_{x x} \hat{X}-K_{x \delta} \delta \\
m \cdot \frac{\partial^{2} \hat{Z}}{\partial t^{2}}=\int_{S_{b}} p \frac{\partial z}{\partial n} \mathrm{~d} s-\rho g \int_{S_{b}} \hat{Z} \frac{\partial z}{\partial n} \mathrm{~d} s-K_{z z} \hat{Z} \\
I \frac{\partial^{2} \delta}{\partial t^{2}}=\int_{S_{b}} p \cdot\left[\left(z-\bar{z}_{0}\right) \frac{\partial x}{\partial n}-\left(x-\bar{x}_{0}\right) \frac{\partial z}{\partial n}\right] \mathrm{d} s \\
-\rho g \int_{S_{b}} \delta\left(x-\bar{x}_{0}\right) \cdot\left[\left(z-\bar{z}_{0}\right) \frac{\partial x}{\partial n}-\left(x-\bar{x}_{0}\right) \frac{\partial z}{\partial n}\right] \mathrm{d} s \\
-K_{\delta x} \hat{X}-K_{\delta \delta} \delta
\end{gathered}
$$

where, $m$ is the mass of the floating structure, $I$ is the mass moment of inertia about the center of the mass of the structure, $p$ is the dynamic pressure of the fluid.

$$
p=-\rho \frac{\partial \Phi}{\partial t}=-i \rho g \varsigma_{0} \varphi e^{i \sigma t}
$$

The mooring system is considered the symmetric fore and aft of the structure. Considering a mooring line $\mathrm{AB}$, with its spring constant $K$ and pretension $F_{0}$, the coordinates of the attachment point on the structure and sea floor are $\left(x_{a}, z_{a}\right)$ and $\left(x_{b}, z_{b}\right)$, respectively. By ignoring the inertia effects of the mooring line and the viscous forces on the line, Weng and Chou [13] derived each component of the forces and moments coefficient by using the mooring system:

$$
\begin{gathered}
K_{x x}=2\left[\frac{\left(x_{b}-x_{a}\right)^{2}}{l_{a b}^{2}} K+\frac{\left(z_{b}-z_{a}\right)^{2}}{l_{a b}^{2}} \frac{F_{0}}{l_{a b}}\right] \\
K_{z z}=2\left[\frac{\left(z_{b}-z_{a}\right)^{2}}{l_{a b}^{2}} K+\frac{\left(x_{b}-x_{a}\right)^{2}}{l_{a b}^{2}} \frac{F_{0}}{l_{a b}}\right] \\
K_{x \delta}=\frac{2}{l_{a b}^{2}}\left[K\left(x_{b}-x_{a}\right)^{2}+\frac{F_{0}}{l_{a b}}\left(z_{b}-z_{a}\right)^{2}\right]\left(z_{a}-\bar{z}_{0}\right) \\
-\frac{2}{l_{a b}^{2}}\left[K-\frac{F_{0}}{l_{a b}}\right]\left(x_{b}-x_{a}\right)\left(z_{b}-z_{a}\right)\left(x_{a}-\bar{x}_{0}\right)=K_{\delta x} \\
K_{\delta \delta}=2 \frac{K}{l_{a b}^{2}}\left\{\left[\left(x_{b}-x_{a}\right)\left(z_{a}-\bar{z}_{b}\right)-\left(z_{b}-z_{a}\right)\left(x_{a}-\bar{x}_{0}\right)\right]^{2}\right. \\
\left.+\left[\left(z_{b}-z_{a}\right)\left(z_{a}-\bar{z}_{b}\right)+\left(x_{b}-x_{a}\right)\left(x_{a}-\bar{x}_{0}\right)\right]^{2} \frac{F_{0}}{l_{a b}}\right\}
\end{gathered}
$$

\subsection{Flexible Skirt Boundary}

The flexible skirt is taken to behave as a one-dimensional beam of uniform flexural rigidity with various kinds of Young's modulus, assuming the zero thickness of the idealized. The equation of the motion of the flexible skirt is acted upon by fluid pressure which may be written in terms of the complex displacement amplitude $v(z)$ as Clough and Penzien [14]:

$$
E I \frac{\partial^{4} v(z)}{\partial z^{4}}-m_{s} \sigma^{2} v(z)=i \rho g \varsigma_{0} \varphi
$$

where, $\rho$ is the fluid density, then the boundary conditions are: 


$$
\begin{aligned}
& v=\frac{\mathrm{d} v}{\mathrm{~d} z}=0 \quad \text { on } \quad z=-q_{1} h \\
& \frac{\mathrm{d}^{2} v}{\mathrm{~d} z^{2}}=0 \quad \text { on } \quad z=-\left(q_{1} h+q_{2} h\right) \\
& \frac{\mathrm{d}^{3} v}{\mathrm{~d} z^{3}}=0 \quad \text { on } \quad z=-\left(q_{1} h+q_{2} h\right)
\end{aligned}
$$

Eq. (22) implies zero displacement, zero slope at the tip of the flexible skirts at $z=-q_{1} h$. Eqs. (23a) and (23b) imply bending moment and shearing force at the tip of the flexible skirts at $z=-\left(q_{1} h+q_{2} h\right)$, respectively.

A suitable Green function $v_{G}\left(z ; z^{\prime}\right)$ for the structural problem satisfies each boundary condition.

$$
\begin{aligned}
& \frac{\mathrm{d}^{4} v_{G}}{\mathrm{~d} z^{4}}-\frac{m_{s} \sigma^{2}}{E I} v_{G}=\delta\left(z-z^{\prime}\right) \\
& \text { on } \quad-\left(q_{1} h+q_{2} h\right) \leq z \leq-q_{1} h \\
& v_{G}=\frac{\mathrm{d} v_{G}}{\mathrm{~d} z}=0 \quad \text { on } \quad z=-q_{1} h \\
& \frac{\mathrm{d}^{2} v_{G}}{\mathrm{~d}^{2}}=0 \quad \text { on } \quad z=-\left(q_{1} h+q_{2} h\right) \\
& \frac{\mathrm{d}^{3} v_{G}}{\mathrm{~d}^{3}}=0 \quad \text { on } \quad z=-\left(q_{1} h+q_{2} h\right)
\end{aligned}
$$

The flexible skirt boundary conditions may be obtained by the method of Laplace transforms, and the complex displacement amplitude $v_{G}$ may be written:

$$
v_{G}(z)=-\frac{i \rho g \zeta_{0}}{E I} \int_{-\left(q_{1} h+q_{2} h\right)}^{-q_{1} h} v_{G}(z ; \xi) \varphi(x, \xi) \mathrm{d} \xi
$$

where

$$
\begin{aligned}
v_{G}\left(z ; z^{\prime}\right)= & \frac{\alpha_{0}}{2 \sqrt{A}}(\cosh \sqrt[4]{A} z-\cos \sqrt[4]{A} z) \\
& +\frac{\beta_{0}}{2 \sqrt{A}}\left(\frac{\sinh \sqrt[4]{A} z}{\sqrt[4]{A}}-\frac{\sin \sqrt[4]{A} z}{\sqrt[4]{A}}\right) \\
& +\frac{1}{2 \sqrt{A}}\left(\frac{\sinh \sqrt[4]{A}\left(z-z^{\prime}\right)}{\sqrt[4]{A}}-\frac{\sin \sqrt[4]{A}\left(z-z^{\prime}\right)}{\sqrt[4]{A}}\right)
\end{aligned}
$$

in which $A=m_{s} \sigma^{2} /(E I)$.

$$
\begin{aligned}
& \alpha_{0}=D^{-1} \cdot \sqrt[4]{A}\left\{\sinh \sqrt[4]{A} z^{\prime}+\sin \sqrt[4]{A} z^{\prime}\right. \\
& +\sinh \sqrt[4]{A}\left(q_{1} h+q_{2} h+z^{\prime}\right) \cos \sqrt[4]{A}\left(q_{1} h+q_{2} h\right) \\
& +\sin \sqrt[4]{A}\left(q_{1} h+q_{2} h+z^{\prime}\right) \cosh \sqrt[4]{A}\left(q_{1} h+q_{2} h\right) \\
& -\cosh \sqrt[4]{A}\left(q_{1} h+q_{2} h+z^{\prime}\right) \sin \sqrt[4]{A}\left(q_{1} h+q_{2} h\right) \\
& \left.-\cos \sqrt[4]{A}\left(q_{1} h+q_{2} h+z^{\prime}\right) \sinh \sqrt[4]{A}\left(q_{1} h+q_{2} h\right)\right\} \\
& \beta_{0}=-\sqrt{A} \cdot D^{-1}\left\{\cosh \sqrt[4]{A} z^{\prime}+\cos \sqrt[4]{A} z^{\prime}\right. \\
& +\cosh \sqrt[4]{A}\left(q_{1} h+q_{2} h\right) \cos \sqrt[4]{A}\left(q_{1} h+q_{2} h+z^{\prime}\right) \\
& +\cos \sqrt[4]{A}\left(q_{1} h+q_{2} h\right) \cosh \sqrt[4]{A}\left(q_{1} h+q_{2} h+z^{\prime}\right) \\
& -\sinh \sqrt[4]{A}\left(q_{1} h+q_{2} h\right) \sin \sqrt[4]{A}\left(q_{1} h+q_{2} h+z^{\prime}\right) \\
& \left.+\sin \sqrt[4]{A}\left(q_{1} h+q_{2} h\right) \sinh \sqrt[4]{A}\left(q_{1} h+q_{2} h+z^{\prime}\right)\right\}
\end{aligned}
$$

and

$$
D=2 \sqrt{A}\left[1+\cosh \sqrt[4]{A}\left(q_{1} h+q_{2} h\right) \cos \sqrt[4]{A}\left(q_{1} h+q_{2} h\right)\right]
$$

\subsection{Floating Structure Boundary Condition}

When a moored floating structure is at rest, the fluid pressure, mooring line tension, buoyancy and mass force should be a balanced state, and taking into consideration the exciting wave and radiation wave by the floating structure's motion. Therefore, when the floating structure is subjected to a train of small amplitude waves, the motion amplitude of the floating structure can be acquired after arranging Eqs. (17)-(19), and the equation can then be simplified to a simpler form. The amplitudes of surge, heave and pitch motion may be expressed as:

$$
\begin{aligned}
& \frac{\xi}{\varsigma_{0}}=\frac{-i}{R}\left\{B_{2} C_{3} \int_{S_{b}} \varphi \frac{\partial x}{\partial n} \mathrm{~d} s\right. \\
& \left.-B_{2} C_{1} \int_{S_{b}} \varphi\left[\left(z-\bar{z}_{0}\right) \frac{\partial x}{\partial n}-\left(x-\bar{x}_{0}\right) \frac{\partial z}{\partial n}\right] \mathrm{d} s\right\} \\
& \frac{\eta}{\varsigma_{0}}=\frac{-i}{R}\left[A_{1} C_{3}-A_{3} C_{1}\right] \int_{S_{b}} \varphi \frac{\partial z}{\partial n} \mathrm{~d} s \\
& \frac{\omega}{\varsigma_{0}}=\frac{-i}{R}\left\{-A_{3} B_{2} \int_{S_{b}} \varphi \frac{\partial x}{\partial n} \mathrm{~d} s\right. \\
& \left.+A_{1} B_{2} \int_{S_{b}} \varphi\left[\left(z-\bar{z}_{0}\right) \frac{\partial x}{\partial n}-\left(x-\bar{x}_{0}\right) \frac{\partial z}{\partial n}\right] \mathrm{d} s\right\}
\end{aligned}
$$


where, $R=B_{2}\left(A_{1} C_{3}-A_{3} C_{1}\right)$ and

$$
\begin{gathered}
A_{1}=\frac{K_{x x}}{\rho g}-\frac{m}{\rho} \frac{\sigma^{2}}{g} ; \\
C_{1}=A_{3}=\frac{K_{x \delta}}{\rho g} ; \\
B_{2}=-\frac{m}{\rho} \frac{\sigma^{2}}{g}+\int_{S_{b}} \frac{\delta z}{\delta n} \mathrm{~d} s+\frac{K_{z z}}{\rho g} ; \\
C_{3}=\frac{K_{\delta \delta}}{\rho g}-\frac{I_{0}}{\rho} \frac{\sigma^{2}}{g} \\
+\int_{S_{b}}\left(x-\bar{x}_{0}\right)\left[\left(z-\bar{z}_{0}\right) \frac{\partial x}{\partial n}-\left(x-\bar{x}_{0}\right) \frac{\partial z}{\partial n}\right] \mathrm{d} s
\end{gathered}
$$

Substituting Eqs. (28)-(30) into Eq. (16), associating with Eq. (15), the potential on the immersed surface of the floating structure with flexible skirts is:

$$
\begin{aligned}
& \frac{\partial \varphi}{\partial n}=\frac{\sigma^{2}}{g R}\left\{\left[B_{2} C_{3} \frac{\partial x}{\partial n} \int_{S_{b}} \varphi \frac{\partial x}{\partial n} \mathrm{~d} s\right.\right. \\
& \left.-B_{2} C_{1} \frac{\partial x}{\partial n} \int_{S_{b}} \varphi\left[\left(z-\bar{z}_{0}\right) \frac{\partial x}{\partial n}-\left(x-\bar{x}_{0}\right) \frac{\partial z}{\partial n}\right] \mathrm{d} s\right] \\
& +\left[A_{1} C_{3}-A_{3} C_{1}\right] \frac{\partial z}{\partial n} \int_{S_{b}} \varphi \frac{\partial z}{\partial n} \mathrm{~d} s+\left[-A_{3} B_{2} \int_{S_{b}} \varphi \frac{\partial x}{\partial n} \mathrm{~d} s\right. \\
& \left.+A_{1} B_{2} \int \varphi\left[\left(z-\bar{z}_{0}\right) \frac{\partial x}{\partial n}-\left(x-\bar{x}_{0}\right) \frac{\partial z}{\partial n}\right] \mathrm{d} s\right] \\
& \cdot\left[\left(z-\bar{z}_{0}\right) \frac{\partial x}{\partial n}-\left(x-\bar{x}_{0}\right) \frac{\partial z}{\partial n}\right] \\
& +\frac{\partial x}{\partial n} \frac{\rho g}{E I} \int_{-\left(q_{1} h+q_{2} h\right)}^{-q_{1} h}\left\{\frac{\alpha_{0}}{2 \sqrt{A}}(\cosh \sqrt[4]{A} z-\cos \sqrt[4]{A} z)\right. \\
& +\frac{\beta_{0}}{2 \sqrt{A}}\left(\frac{\sinh \sqrt[4]{A} z}{\sqrt[4]{A}}-\frac{\sin \sqrt[4]{A} z}{\sqrt[4]{A}}\right) \\
& \left.\left.+\frac{1}{2 \sqrt{A}}\left(\frac{\sinh \sqrt[4]{A}\left(z-z^{\prime}\right)}{\sqrt[4]{A}}-\frac{\sin \sqrt[4]{A}\left(z-z^{\prime}\right)}{\sqrt[4]{A}}\right) \varphi \frac{\partial x}{\partial n} \mathrm{~d} z^{\prime}\right\}\right\}
\end{aligned}
$$

\subsection{Forces on Mooring Lines}

For a mooring line in Fig. 1, its coordinate of attachment point on structure is transferred from $\left(x_{a}, z_{a}\right)$ to $\left(x_{a}^{\prime}, z_{a}^{\prime}\right)$ when structure is oscillated in response to fluid forces. Forces on the mooring line can be estimated easily from lengthening $\varepsilon$ and spring constant $K_{a b}$ of mooring line $\left(F_{a b}=K_{a b} \cdot \varepsilon\right)$. The lengthening $\varepsilon$ can be written as:

$$
\begin{aligned}
& \varepsilon=\frac{-1}{l_{a b}}\left\{\left(x_{b}-x_{a}\right)\left(x_{0}-\bar{x}_{0}\right)+\left(z_{b}-z_{a}\right)\left(z_{0}-\bar{z}_{0}\right)\right. \\
& \left.+\left[\left(x_{b}-x_{a}\right)\left(z_{a}-\bar{z}_{0}\right)-\left(z_{b}-z_{a}\right)\left(x_{a}-\bar{x}_{0}\right)\right] \delta\right\}
\end{aligned}
$$

Thus, when mooring lines are treated as a linear system, and is expressed as:

$$
\begin{aligned}
& \frac{F_{a b}}{K_{a b} \varsigma_{0}}=\frac{-1}{l_{a b}}\left\{\left(x_{b}-x_{a}\right) \frac{x_{0}-\bar{x}_{0}}{\varsigma_{0}}+\left(z_{b}-z_{a}\right) \frac{z_{0}-\bar{z}_{0}}{\varsigma_{0}}\right. \\
& \left.+\left[\left(x_{b}-x_{a}\right)\left(z_{a}-\bar{z}_{0}\right)-\left(z_{b}-z_{a}\right)\left(x_{a}-\bar{x}_{0}\right)\right] \frac{\delta}{\varsigma_{0}}\right\}
\end{aligned}
$$

where, $l_{a b}=\sqrt{\left(x_{b}-x_{a}\right)^{2}+\left(z_{b}-z_{a}\right)^{2}}$.

\subsection{Coefficients of Reflection and Transmission}

The coefficients of reflection and transmission are determined using the continuity of mass and energy flux on the fictitious boundaries. Substituting Eq. (6) into Eq. (10), multiplying with $\cosh k(h+z)$, and integrating from $z=-h$ to $z=0$, the reflection coefficient, $K_{r}$, can be expressed in terms of the normal derivatives of potential, $\bar{\varphi}_{1}$, as:

$$
\begin{array}{r}
K_{r}=1+\frac{i}{N_{0} \sinh k h} \int_{-h}^{0} \bar{\varphi}_{1} \cosh k(h+z) \mathrm{d} z \\
\text { on } x=\ell_{1}
\end{array}
$$

where, $N_{0}=(1+2 k h / \sinh 2 k h) / 2$.

Substituting Eq. (33) into Eq. (5), associating with Eq. (9), the relationship between the potential of the auxiliary boundary $x=\ell_{1}$, and its normal derivative can be written as:

$$
\begin{aligned}
& \varphi\left(\ell_{1}, z\right)=2 \frac{\cosh k(h+z)}{\cosh k h} \\
& +2 i \frac{\cosh k(h+z)}{N_{0} \sinh 2 k h} \int_{-h}^{0} \bar{\varphi}\left(\ell_{1}, z\right) \cosh k(h+z) \mathrm{d} s
\end{aligned}
$$

Similarly, the coefficient of transmission can be 
derived by substituting Eq. (8) into Eq. (12), multiplying with $\cosh k(h+z)$, and integrating from $z=-h$ to $z=0$, to yield the following form:

$$
\begin{array}{r}
K_{t}=\frac{i}{N_{0} \sinh k h} \int_{-h}^{0} \bar{\varphi}_{2} \cosh k(h+z) \mathrm{d} z \\
\text { on } x=-\ell_{2}
\end{array}
$$

The relationship between the potential and its normal derivative on the auxiliary boundary, $x=-\ell_{2}$, can be obtained by substituting Eq. (35) into Eq. (7), and associating with Eq. (11):

$$
\begin{aligned}
& \varphi\left(-\ell_{2}, z\right)=2 i \frac{\cosh k(h+z)}{N_{0} \sinh 2 k h} \\
& \cdot \int_{-h}^{0} \bar{\varphi}\left(-\ell_{2}, z\right) \cosh k(h+z) \mathrm{d} s
\end{aligned}
$$

\subsection{Dual Boundary Element Method}

The above problem for the fluid potential of Region II is solved numerically by using the dual boundary element method. According to Green's second identity law, the first equation of the dual boundary element method for the potential of any point on the boundaries of Region II is subject to the potential on the boundaries combined with its first normal derivative:

$$
\pi \varphi\left(\xi^{\prime}, \eta^{\prime}\right)=\int_{\Gamma_{2}}\left[\frac{\partial \varphi(\xi, \eta)}{\partial n_{x z}}\left(\ln \frac{1}{r}\right)-\varphi(\xi, \eta) \frac{\partial}{\partial n_{x z}}\left(\ln \frac{1}{r}\right) \mathrm{d} s\right.
$$

where, $\ln 1 / r$ is the solution of Laplace equation. $n_{x z}$ is the normal vector of point $(\xi, \eta)$. When the boundaries enclosing Region II are partitioned into $N$ segments, Eq. (37) indicates a matrix form as follows.

$$
\left[G_{i j}\right]\left\{\varphi_{i}\right\}=\left[H_{i j}\right]\left\{\frac{\partial \varphi}{\partial n_{j}}\right\} \quad(i, j=1,2, \ldots, N)
$$

The second equation of the dual boundary integral formulation for the points on the boundaries is derived as:

$$
\begin{aligned}
& \pi \frac{\partial \varphi\left(\xi^{\prime}, \eta^{\prime}\right)}{\partial n_{\overline{x z}}}= \\
& \int_{\Gamma_{2}}\left[\frac{\partial \varphi(\xi, \eta)}{\partial n_{x z}} \frac{\partial}{\partial n_{\overline{x z}}}\left(\ln \frac{1}{r}\right)-\varphi(\xi, \eta) \frac{\partial^{2}}{\partial n_{x z} \partial n_{\overline{x z}}}\left(\ln \frac{1}{r}\right)\right] \mathrm{d} s
\end{aligned}
$$

where, $n_{\overline{x z}}$ is the normal vector of point $\left(\xi^{\prime}, \eta^{\prime}\right)$. Eq. (39) also displays a matrix form:

$$
\left[\bar{G}_{i j}\right]\left\{\varphi_{i}\right\}=\left[\bar{H}_{i j}\right]\left\{\frac{\partial \varphi}{\partial n_{j}}\right\} \quad(i, j=1,2, \ldots, N)
$$

Matrices of Eq. (38) and Eq. (40) are dependent on the geography of the analytical domain and have been derived by Chen [15]. Boundaries of the flexible skirts of the floating structure are degenerate boundaries since the thicknesses of the flexible skirts are assumed to be zero. Eqs. (38) and (40) are a linearly dependent equation and cannot be solved directly. However, the degenerated system can be desingularized by rearranging the matrix of degenerate boundaries between Eqs. (38) and Eq. (40), and formulating a set of new independent equations as follows:

$$
\left[G_{i j}^{*}\right]\left\{\varphi_{i}\right\}=\left[H_{i j}^{*}\right]\left\{\frac{\partial \varphi}{\partial n_{j}}\right\} \quad(i, j=1,2, \ldots, N)
$$

Substituting the boundary conditions of Region II, and Eqs. (13), (14), (34), (36) and (31), into Eq. (41), the potential and its normal derivative on the boundaries of Region II can be obtained. The motion amplitude of each mode and the coefficients of reflection and transmission can also be acquired by substituting the potential on the immersed surface of the structure into Eqs. (28)-(30), (33) and (35).

\section{Results and Discussions}

\subsection{Verification of Numerical Model}

The efficiency of the numerical model in simulating free body dynamics with or without rigid skirts was verified by comparing with the results of Gesraha [11]. As shown in Fig. 2, a good agreement between calculated and Gesraha results are clearly observed over the whole range of the dimensionless parameter $k a$. It is obvious that, the natural period of the structure's heave motion tends to increase with the length of the skirts.

\subsection{Influence of Skirt's Rigidity on Structure's Motions}

The RAOs in surge, heave and pitch of the moored 


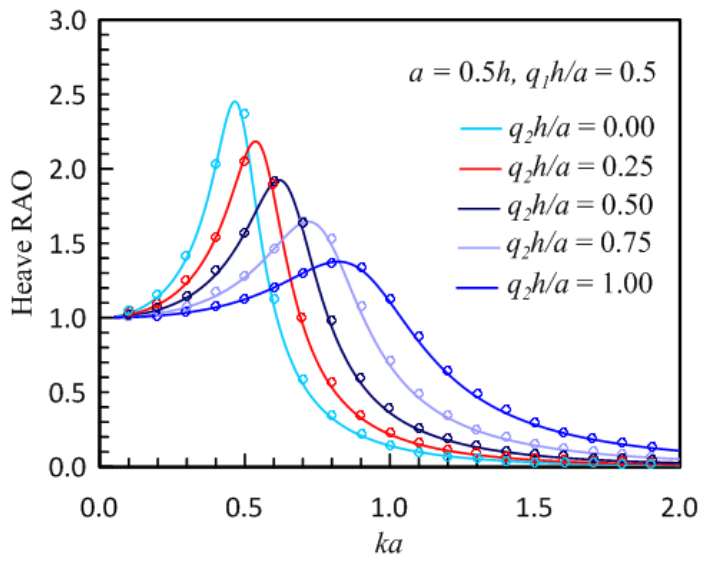

Fig. 2 Comparison between simulated and Gesraha results heave RAO with $a=0.5 h$ and $q_{1} h / a=0.5$ (line: present results, dot: Gesraha results).

floating structure as a function of the dimensionless parameter $k a$ were analyzed for various flexible rigidities of skirt $\left(E I / \rho g h^{5}=0.001,0.050,0.500\right.$, 5.000 and rigid case). The floating structure, with its draft $q_{1}=0.2$, width $h=2 a$ and center of mass $\bar{z}_{0}=-q_{1} h / 2$, is moored by taut springs and prepared for simulation. Each spring has constant stiffness $K / \rho g h=0.009$, pretension force $F_{0} / \rho g h^{2}=0.0115$ and an incline of $60^{\circ}$ to the horizontal bottom.

The motion responses of the floating structure with skirts of $q_{2}=0.3$ are shown in Figs. 3-5. These figures also present the RAOs for the floating structure without skirts $\left(q_{2}=0.0\right)$. The results illustrate that, the

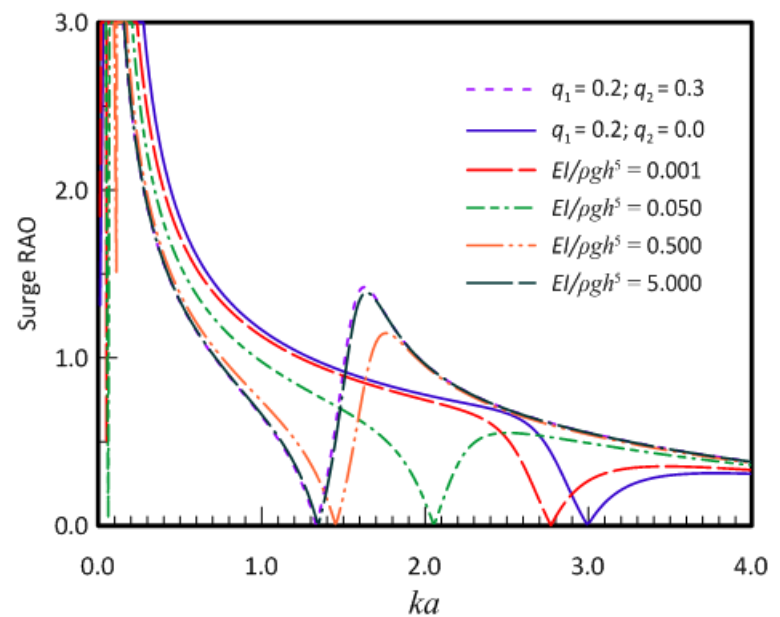

Fig. 3 Influence of the skirt's rigidity $\left(E I / \rho g h^{5}=0.001\right.$, 0.050, 0.500, 5.000 and rigid case) on the surge RAO of the moored floating structure $\left(h=2 a, q_{1}=0.2\right.$ and $q_{2}=0.3 ; q_{2}=$ 0.0 for the case without skirts).

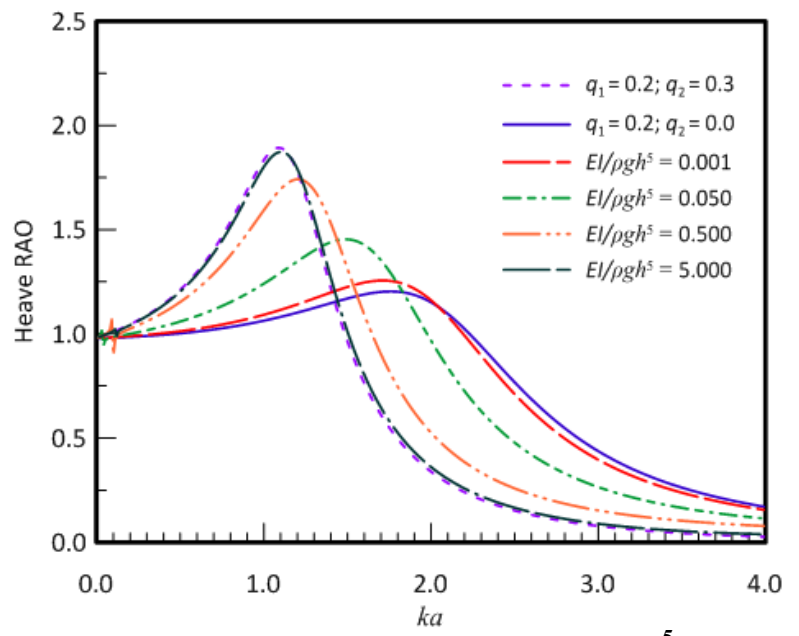

Fig. 4 Influence of the skirt's rigidity $\left(E I / \rho g h^{5}=0.001\right.$, $0.050,0.500,5.000$ and rigid case) on the heave RAO of the moored floating structure $\left(h=2 a, q_{1}=0.2\right.$ and $q_{2}=0.3 ; q_{2}=$ 0.0 for the case without skirts).

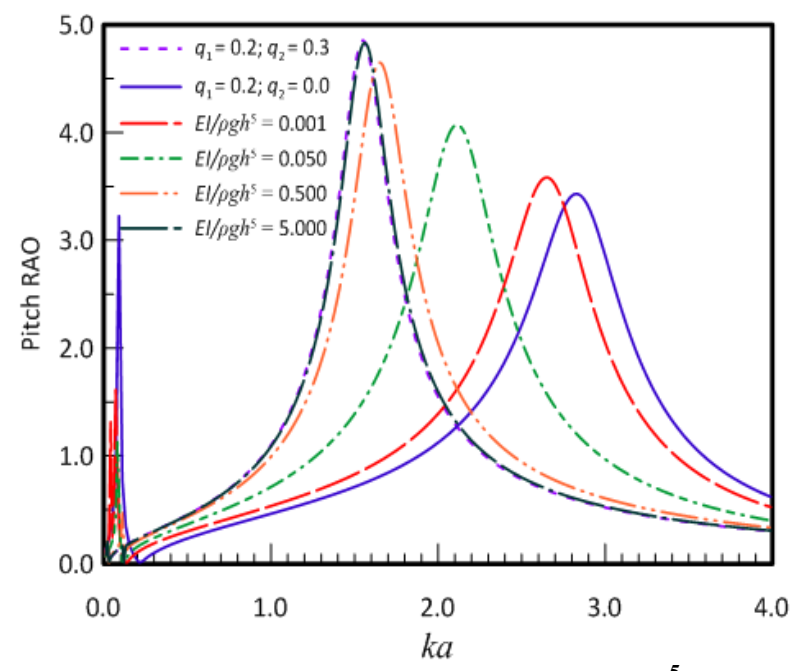

Fig. 5 Influence of the skirt's rigidity $\left(E I / \rho g h^{5}=0.001\right.$, 0.050, 0.500, 5.000 and rigid case) on the pitch RAO of the moored floating structure $\left(h=2 a, q_{1}=0.2\right.$ and $q_{2}=0.3 ; q_{2}=$ 0.0 for the case without skirts).

modulations of surge, heave and pitch responses of the floating structure with the skirt's rigidity $E I / \rho g h^{5}$ $=5.000$ are very closed to the results of rigid skirts. Moreover, the results of the floating structure with skirt's rigidity $E I / \rho g h^{5}=0.001$ approach to the case without skirts. As the flexible rigidity of skirt gradually decreases, the natural frequency of structure's oscillation tends to higher region of $k a$, and the oscillation values in each mode decrease in range as the natural frequency approached. 


\subsection{The Wave Reflection and Transmission Coefficients}

Figs. 6 and 7 show the reflection and transmission coefficients versus $k a$ when the skirt's rigidities $\left(E I / \rho g h^{5}\right)$ are $0.001,0.050,0.500,5.000$ as well as rigid case. The results for the moored floating structure without skirts $\left(q_{2}=0.0\right)$ are also presented in Figs. 6 and 7. In general, the reflection coefficient increases and transmission coefficient decreases as the wave period decreases. However, the resonance of the moored floating structure causes a large motion corresponding to the structure's natural frequency in each mode of motion, which enhances the wave disturbing ability of the structure and has a profound effect on both the wave reflection and transmission coefficients. In addition, when the thin skirts change their flexible rigidity from 5.000 to 0.001 , the natural frequency of the structure inclines to higher region of $k a$ and toward the reflection curve without skirts.

\subsection{The Influences of Mooring Line Forces}

The floating structure has two mooring lines and the forces on them can be obtained by using Eq. (32). Figs. 8 and 9 present the forces on mooring lines of aft and fore side, respectively. The forces on mooring lines depend on the RAOs of surge, heave and pitch motion.

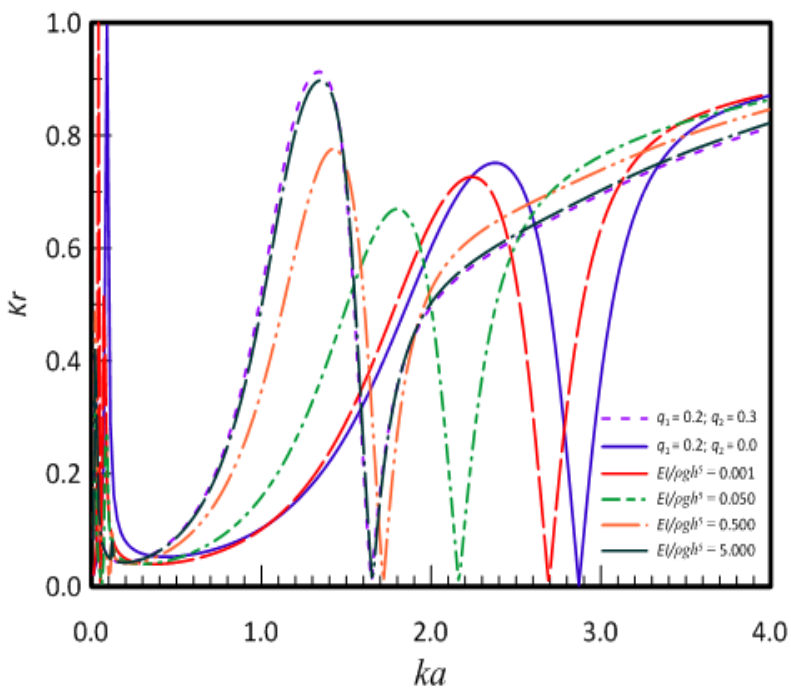

Fig. 6 Correlation between wave reflection coefficient and $k a$ for different skirt's rigidities $\left(E I / \rho g h^{5}=0.001,0.050,0.500\right.$, 5.000 and rigid case) of the moored floating structure $(h=2 a$, $q_{1}=0.2$ and $q_{2}=0.3 ; q_{2}=0.0$ for the case without skirts).

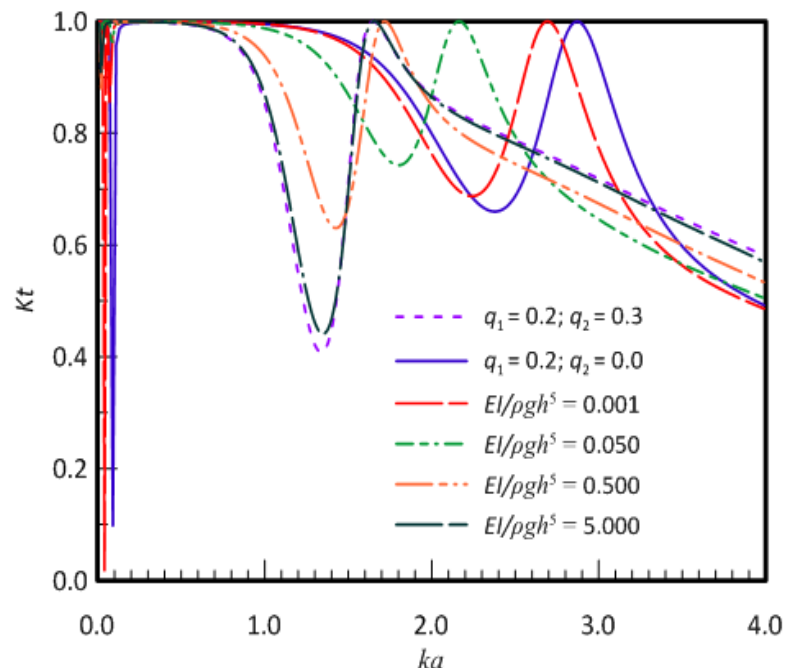

Fig. 7 Correlation between wave transmission coefficient and $\boldsymbol{k} a$ for different skirt's rigidities $\left(E I / \rho g h^{5}=0.001,0.050\right.$, $0.500,5.000$ and rigid case) of the moored floating structure ( $h=2 a, q_{1}=0.2$ and $q_{2}=0.3 ; q_{2}=0.0$ for the case without skirts).

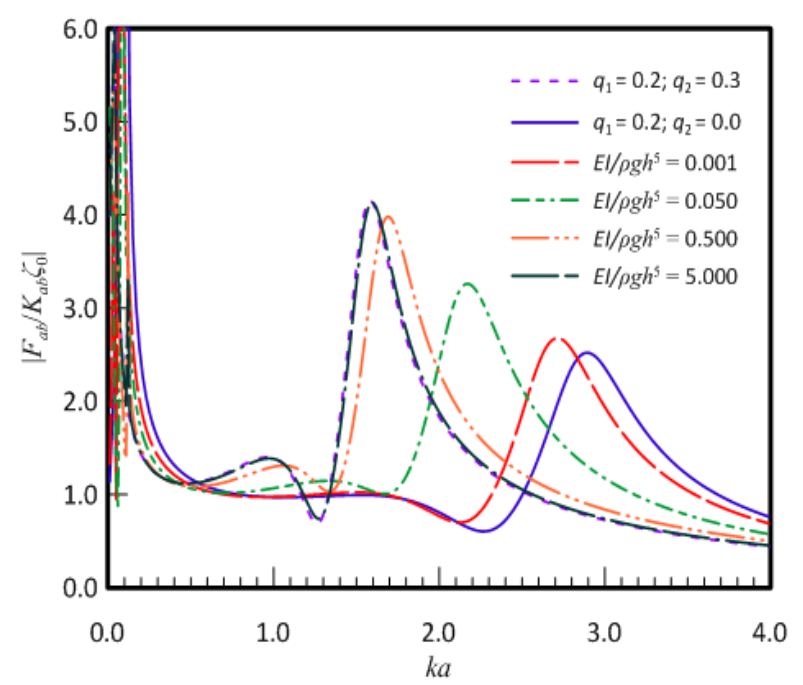

Fig. 8 Correlation between mooring line forces (fore side) and $k a$ for different skirt's rigidities $\left(E I / \rho g h^{5}=0.001,0.050\right.$, $0.500,5.000$ and rigid case) of the moored floating structure ( $h=2 a, q_{1}=0.2$ and $q_{2}=0.3 ; q_{2}=0.0$ for the case without skirts).

Similar curve trends also occurred in the mooring forces, i.e., the natural frequency of mooring force tends to higher region of $k a$ as the flexible rigidity of skirt gradually decreases. The high peak of the mooring force gradually decreases in value as the flexible rigidity decreases from 5.000 to 0.001 . It is noticed that, the mooring force at aft side (Fig. 8) positively correlates with the pitch RAO of the floating structure (Fig. 5). 


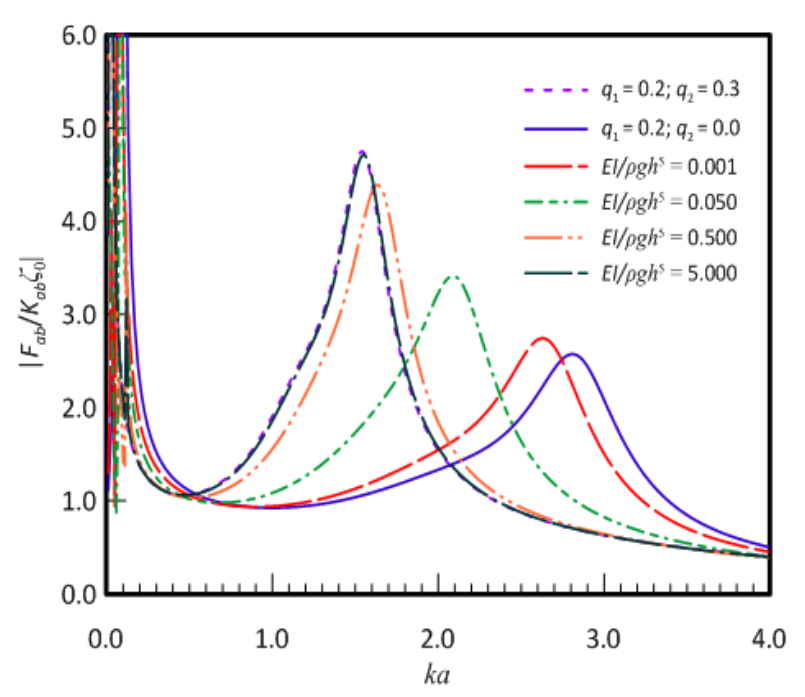

Fig. 9 Correlation between mooring line forces (aft side) and $k a$ for different skirt's rigidities $\left(E I / \rho g h^{5}=0.001,0.050\right.$, 0.500, 5.000 and rigid case) of the moored floating structure ( $h=2 a, q_{1}=0.2$ and $q_{2}=0.3 ; q_{2}=0.0$ for the case without skirts).

\section{Conclusions}

In this study, a numerical model has been developed through usage of the DBEM to analyze the dynamic behavior of a floating structure with flexible skirts. The thickness of the flexible skirt was assumed to be zero. Discussions focused on the influence of the skirt rigidity on structural motion, reflection and transmission coefficients, and mooring line forces. The conclusions can be summarized as: (1) The numerical model is capable of analyzing the motions of a moored floating structure with flexible and extremely thin skirts attached to the structure's bottom, and its validity was verified by comparing the analytical results for rigid skirts. (2) The flexible rigidity of skirts is a critical factor affecting the natural frequency of structure's oscillation as well as moored force, wave reflection and transmission. As the flexible rigidity gradually decreases, the natural frequencies of structure's oscillation, moored force, wave reflection and transmission tend to the region of short-period waves, and the oscillation values of three RAOs and moored force decrease as the natural frequency approached. In addition, the mooring force at aft side positively correlates with the pitch RAO of the floating structure.

\section{References}

[1] Murali, and Mani, J. 1997. "Performance of Cage Floating Breakwater." Journal of Waterway, Port, Coastal, and Ocean Engineering 123 (4): 172-9.

[2] Isaacson, M., and Byres, R. 1988. "Floating Breakwater Response to Wave Action." In Proceedings of the Coastal Engineering Proceedings, 2189-200.

[3] Sannasiraj, S., Sundar, V., and Sundaravadivelu, R. 1998. "Mooring Forces and Motion Responses of Pontoon-Type Floating Breakwaters." Ocean Engineering 25 (1): 27-48.

[4] Sannasiraj, S., Sundaravadivelu, R., and Sundar, V. 2000. "Diffraction-Radiation of Multiple Floating Structures in Directional Waves." Ocean Engineering 28 (2): 201-34.

[5] Lee, J., and Cho, W. 2003. "Hydrodynamic Analysis of Wave Interactions with a Moored Floating Breakwater Using the Element-Free Galerkin Method." Canadian Journal of Civil Engineering 30 (4): 720-33.

[6] Kashiwagi, M. 2005. "Wave-Induced Motions of a Body Floating in a Two-Layer Fluid." In Proceedings of the Fiffteenth (2005) International Offshore and Polar Engineering Conference, 358-65.

[7] Huang, C. C., and Tang, H. J. 2009. "Dynamic Responses of Moored Floating Dual Pontoon Structure in a Fully Nonlinear Numerical Wave Tank." In Proceedings of the Nineteenth (2009) International Offshore and Polar Engineering Conderence, 414-21.

[8] Wang, H. Y., and Sun, Z. C. 2010. "Experimental Study on the Influence of Geometrical Configuration of Porous Floating Breakwater on Performance." Journal of Marine Science and Technology 18 (4): 574-9.

[9] Williams, P. G., and McDougal, W. 1991. "Flexible Floating Breakwater." Journal of Waterway, Port, Coastal, and Ocean Engineering 117 (5): 429-50.

[10] Williams, P. G., and McDougal, W. 1992. "A Submerged Compliant Breakwater." Journal of Offshore Mechanics and Arctic Engineering 114 (2): 83-90.

[11] Gesraha, M. R. 2006. "Analysis of П Shaped Floating Breakwater in Oblique Waves: I. Impervious Rigid Wave Boards." Applied Ocean Research 28 (5): 327-38.

[12] Wang, K. H., and Ren, X. 1993. "Water Waves on Flexible and Porous Breakwaters." Journal of Engineering Mechanics 119 (5): 1025-47.

[13] Weng, W. K., and Chou, C. R. 2007. "Analysis of Responses of Floating Dual Pontoon Structure." China Ocean Engineering 21 (1): 91-104.

[14] Clough, R. W., and Penzien, J. 1993. Dynamics of Structures. New York: McGraw-Hill.

[15] Chen, J. T., and Hong, H. K. 1983. Boundary Element Method. Taipei: New-World. 